\title{
Appetite regulation: new opportunities for weight control
}

\author{
Dave Morgan \\ Department of Metabolic Medicine, Imperial College School of Medicine, Hammersmith Campus, \\ Du-Cane Road, London W12 ONN, UK
}

The increase in obesity and its associated complications of diabetes, heart disease and hypertension is one of the greatest health problems facing the Western world. An understanding of the mechanisms regulating food intake, energy expenditure and energy balance is important for combating this epidemic. The hypothalamus is the crucial brain area controlling food intake. Initial studies in the first half of this century identified anatomicallydistinct feeding and satiety centres. The system became more complex with the discovery of neuropeptides with both orexigenic and anorectic actions. However, the regions initially identified do appear to be important foci for these neuropeptide systems. The arcuate nucleus and the lateral hypothalamus (LH) increasingly appear important.

The LH was identified as a 'feeding centre' following the observation that lesions of this area in the rat led to loss of appetite, anorexia and starvation. This idea was criticised, as the LH is a diffuse nucleus with few distinct chemical markers, through which many fibres pass. Two novel neuropeptides, which both alter food intake, provide a chemical signature, allowing investigation of this area. Melanin-concentrating hormone is expressed only in the $\mathrm{LH}$, and its expression is regulated by energy balance. When injected intracerebrally into rats it increases appetite, and mice with null mutations in the melanin-concentrating hormone gene are thin and have reduced food intake. Orexin $\mathrm{A}$ and $\mathrm{B}$ are products of a single gene expressed only in the LH. Again, expression of this gene is increased by fasting, and intracerebral injection increases food intake. Subsequent studies suggest it may be more important in general arousal.
Lesions of the arcuate nucleus cause an opposite syndrome to those of the LH, with hyperphagia and obesity resulting. The arcuate nucleus may be an important point of communication between the circulation and the hypothalamic food intake-regulating mechanisms, as it has a reduced blood-brain barrier. It forms an important focus for both orexigenic and anorectic neuropeptide systems, including: neuropeptide $\mathrm{Y}$ the most powerful orexigenic peptide known; the melanocortin system, which includes both the anorectic peptide $\alpha$-melanocyte-stimulating hormone and its orexigenic endogenous antagonist agouti gene-related protein; the novel anorectic neuropeptide cocaine- and amphetamine-regulated transcript. The arcuate nucleus contains different cell types either expressing both orexigenic peptides (i.e. neuropeptide and agouti gene-related protein) or both anorectic peptides (i.e. $\alpha$-melanocyte-stimulating hormone and cocaine- and amphetamine-regulated transcript). The circulating adipostat hormone leptin binds to receptors on both anorectic and orexigenic cell types, but only activates the anorectic cells.

A complex set of interactions occur between the neuropeptide systems described earlier. These interactions involve both intranuclear connections (e.g. between orexigenic and anorectic cells in the arcuate nucleus, and internuclear connection (e.g. between both arcuate nucleus cell types and both melanin-concentrating hormone and orexin cells in the LH). Other nuclei both within and outside the hypothalamus also have important roles, but the discovery of these peptide systems, and our increasing ability to analyse the dynamics of the interactions between then, is bringing us closer to an understanding of the hypothalamic control of food intake.

\section{Appetite regulation: Lateral hypothalamus: Neuropeptides}

